\title{
ФИЗИКО-МАТЕМАТИЧЕСКОЕ МОДЕЛИРОВАНИЕ
}

\author{
УДК 519.673:532.543
}

Алибеков Г.А.

МАТЕМАТИЧЕСКОЕ МОДЕЛИРОВАНИЕ
ДЛИНЫ ГИДРАВЛИЧЕСКИХ КАНАЛОВ В ЕСТЕСТВЕННОМ
РУСЛЕ С ПОМОЩЬЮ ЭВМ

Alibekov G.A.

\section{MATHEMATICAL MODELING OF THE MAXIMUM LENGHT OF HYDRAULIC CHANNEL IN A NATURAL WATERCOURSE VIA COMPUTERS}

Работа посвящена нахождению максимально допустимой длины гидравлических каналов, проходящих в естественном русле, для предложенного значения параметра коэффициента полезного действия и иных показателях параметров полигонального сечения канала, с применением комплекса программ на ЭВМ.

Ключевые слова: моделирование гидравлических каналов, полигональное сечение канала, максимальная длина канала.

The present paper is devoted to finding the maximum possible length of hydraulic passages extending in a natural line for the given parameters of useful effect coefficient and other performance parameters of polygonal section of the channel, using a set of computer programs.

Key words: modeling of hydraulic channels, polygonal cross-section of the channel, the maximum length of the channel.

\section{Введение}

По причине нехватки водных ресурсов на сельскохозяйственных объектах, часто стоит вопрос о прокладке гидравлических каналов к объектам орошения, с целью увеличения плодоносности различных культур. Для уменьшения финансовых затрат, выгоднее использовать каналы в естественных руслах, то есть без дополнительной облицовки. Определение максимальной длины, на которую дойдет необходимый объем воды по данному типу канала, при заданном коэффициенте полезного действия, является актуальным вопросом, предлагаемым для рассмотрения в работе. Также актуальностью работы является необходимость внедрения языков программирования высокого уровня, с созданием приложения под совре- 
менные операционные системы (Windows), в виду невозможности или высокой трудоемкости при ручном счете.

Цель работы:

- нахождение максимальной длины несущего отрезка гидравлического канала полигонального сечения в естественных грунтах с требуемым коэффициентом полезного действия канала, с учетом потерь воды на фильтрацию и испарение в процессе транспортировки;

- создание прикладного программного обеспечения для моделирования канала полигонального сечения с целью поиска предельно допустимой длины русла.

В основу расчета взят канал полигонального сечения в виду того что, параметры данного сечения канала наилучшим способом соответствуют конфигурациям равноустойчивых откосов, определяемых по методу, основанному на рассмотрении состояния предельного равновесия. Поперечное сечение указанного типа канала приближается по своей форме к естественным руслам, имеющим сечения весьма близкие к параболическим [1].

Коэффициент полезного действия $\mathrm{E}_{\mathrm{b}}$ магистрального канала, распределителя, оросителя или их участков определяется как отношение максимального расхода воды $Q_{\mathrm{nt}}$, забираемого из канала, к максимальному расходу воды $Q_{\mathrm{br}}$, в начале канала с учетом потерь воды на фильтрацию и испарение по его трассе. Коэффициенты полезного действия магистрального канала, его ветвей должны быть не менее 0,90 [2].

В коротких гидравлических каналах значением коэффициента полезного действия(КПД) часто можно пренебречь, в виду незначительных потерь на фильтрацию, а также испарение воды в канале, однако при моделировании больших оросительных каналов, с большой протяженностью, КПД является одним из ключевых параметров. Возникает вопрос: какова максимально допустимая длина гидравлического канала в естественным руслом, при заданном КПД.

Пусть даны следующие параметры канала: тип поперечного сечения канала - полигональный; коэффициенты заложения откосов $m_{1}=2, m_{2}=$ 1,5 ; коэффициент шероховатости $n=0,0225$; продольный уклон, равный уклону местности, $i=0,0005$; расход канала $Q=10 \mathrm{~m}^{3} /$ с. Фильтрационные потери берем как установившиеся и свободные, а работу канала постоянной. Расчет фильтрационных потерь из каналов непрерывного действия в естественном русле при установившейся свободной фильтрации для каналов полигональной формы по следующей зависимости[2]:

$$
Q_{f}=0,0116 k_{\phi}(B+2 h),
$$

где $Q_{f}$ - расход фильтрационных потерь, м $^{3} / \mathrm{c}$, на 1 км длины канала $k_{\phi}-$ коэффициент фильтрации, $B$ - ширина живого сечения поверху, 
$h$ - глубина воды в канале,

Потери воды на испарение [3]:

$$
Q_{u c n}=B \cdot e \cdot l
$$

где $l$ - длина канала,

$e$ - слой испарения с водной поверхности в единицу времени, принимается по данным наблюдений, в работе для условий Республики Дагестан принято $e=10 \mathrm{Mм} /$ сут [4].

Параметры величины избытка бровки магистрального канала без облицовки над предельным показателем среза воды из [2]для упрощения создания программы на ЭВМ линеаризованы выражением[4]:

$$
\Delta=0,2+0,008 Q-0,00004 Q^{2}
$$

Для разработки программы на компьютере был взят язык с++, a peaлизована программа в среде C++ Builder, в виду того что данный язык программирования является языком высокого уровня, а выбранная среда позволяет создавать готовые приложения формата .ехе под современные операционные системы (Windows, Mac OS, Linux и др.)[5].

Основная расчетная часть текста программы DlinaKanala.exe для расчета предельной длины канала:

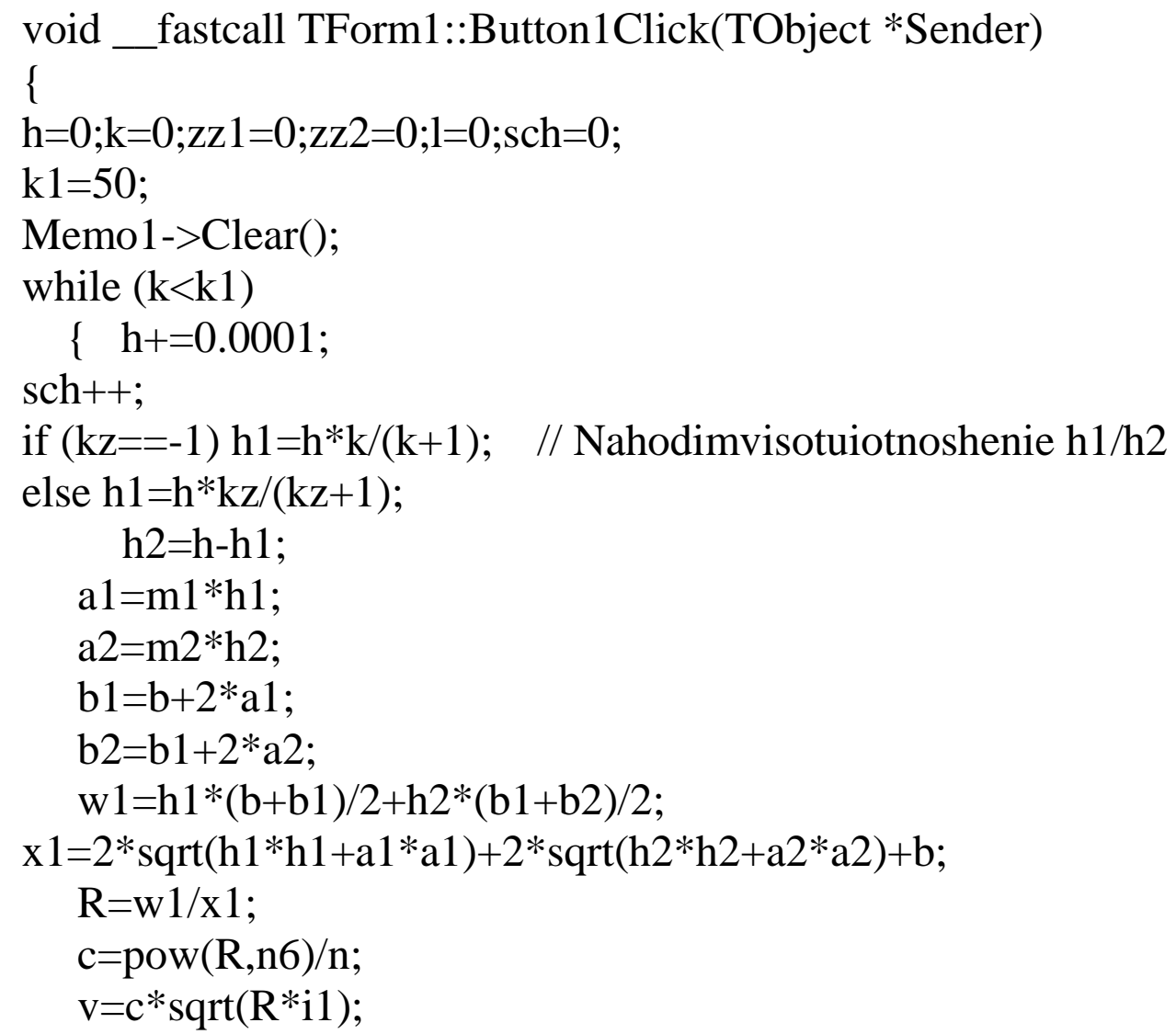




$$
\mathrm{Q}=\mathrm{w} 1{ }^{*} \mathrm{v}
$$

delta $=0.2+0.008 * \mathrm{Q}-0.00004 * \mathrm{Q} * \mathrm{Q}$;

$\mathrm{a} 3=\mathrm{m} 2 *$ delta;

$\mathrm{b} 3=\mathrm{b} 2+2 * \mathrm{a} 3$;

wdelta $=$ delta $*(\mathrm{~b} 2 / 2+\mathrm{b} 3 / 2)$;

$\mathrm{w}=\mathrm{w} 1+$ wdelta; // Obshayaviemka

if $\left(\mathrm{Q}^{*} \mathrm{kpd}>\mathrm{Qz}\right)$

$\{$ if $(\mathrm{zz} 1==0)\{\mathrm{wx}=\mathrm{w} ; \mathrm{zz} 1=1 ;\}$

if $(w x>=w)$

$$
\{\mathrm{hx}=\mathrm{h} ; \mathrm{wx}=\mathrm{w} ; \mathrm{kx}=\mathrm{k} ; \mathrm{Qx}=\mathrm{Q} ; \mathrm{vx}=\mathrm{v} ; \mathrm{xx}=\mathrm{x} 1 ;\}
$$

$\mathrm{h}=0$;

if $(\mathrm{k}==0) \mathrm{k}=0.01$;

if $(\mathrm{kz} !=-1)\{\mathrm{k}=\mathrm{k} 1-0.001 ; \mathrm{kx}=\mathrm{kz} ;\}$

$\mathrm{k}+=\mathrm{k} / 5$;

\}

\}

while $(\mathrm{zz} 2==0)$

$\{1+=0.001$;

$\mathrm{Qr}=0.0116 * \mathrm{kf} *(\mathrm{~b} 2+2 * \mathrm{~h}) * 1+\mathrm{b} 2 * \mathrm{kisp} * 1 / 86400$;

if $(\mathrm{Qz}>(\mathrm{Qx}-\mathrm{Qr}))$

$\{\mathrm{zz} 2=1$;

Series1->Add(kpd,1,clRed);

\}

\}

Memo1 ->Lines->Add("h = " + FloatToStr(hx));

Memo1 ->Lines->Add("k = " + FloatToStr(kx));

Memo1 ->Lines->Add("w = " + FloatToStr(xx));

Memo1 ->Lines->Add("W = " + FloatToStr(wx));

Memo1 ->Lines->Add("Q = " + FloatToStr(Qx*kpd));

Memo1 ->Lines->Add("v = " + FloatToStr(vx));

Memo1 ->Lines->Add("Qnetto = " + FloatToStr(Qx));

Memo1 ->Lines->Add("Qpoter = " + FloatToStr(Qr));

Memo1 ->Lines->Add("Max dlinakanala = " + FloatToStr(1)); \}

Если требуется определить глубину наполнения канала при заданном расходе, задачу необходимо решать методом подбора[6]. Тот же принцип применен при разработке программы для поиска максимальной длины оросительного канала в естественном русле. Для получения более точных данных, шаг для последующего увеличения высоты канала задан равным 0,0001 м, а шаг для последующего увеличения длина канала равный 1м.

Как видно на рисунке 1, после ввода необходимых входных параметров мы получаем подробный расчет всех основных параметров канала, та- 
кие как высота канала, объем выемки грунта, скорость воды в канале, расход брутто и, безусловно, саму предельно допустимую длину канала для заданного значения КПД. Программа считает параметры с высокой точностью, более 5 знаков после запятой. Кроме того, количество выполненных расчетов в программе превышает значения $10^{4}$, что является крайне трудоемким для привычного режима ручного счета моделей каналов. В программе также можно не задаваться значением коэффициента $\mathrm{k}$, определяющего отношение высоты $\mathrm{h}_{1} \mathrm{\kappa} \mathrm{h}_{2}$, в этом случае программа сама даст оптимальные значения параметров $\mathrm{h}_{1}$ и $\mathrm{h}_{2}$, что является еще одним облегчением для проектирования с целью определения наименьшей выемки грунта. Этот фактор также скажется на стоимости канала, т.к. чем меньше грунта выкапывать, тем выгоднее. Программа также выводит графики зависимостей, выбираемые в процессе проектирования пользователем для большей наглядности. Параметры для графика пользователь может выбирать самостоятельно и отслеживать каждый параметр индивидуально, с целью подробного исследования различных моделей.

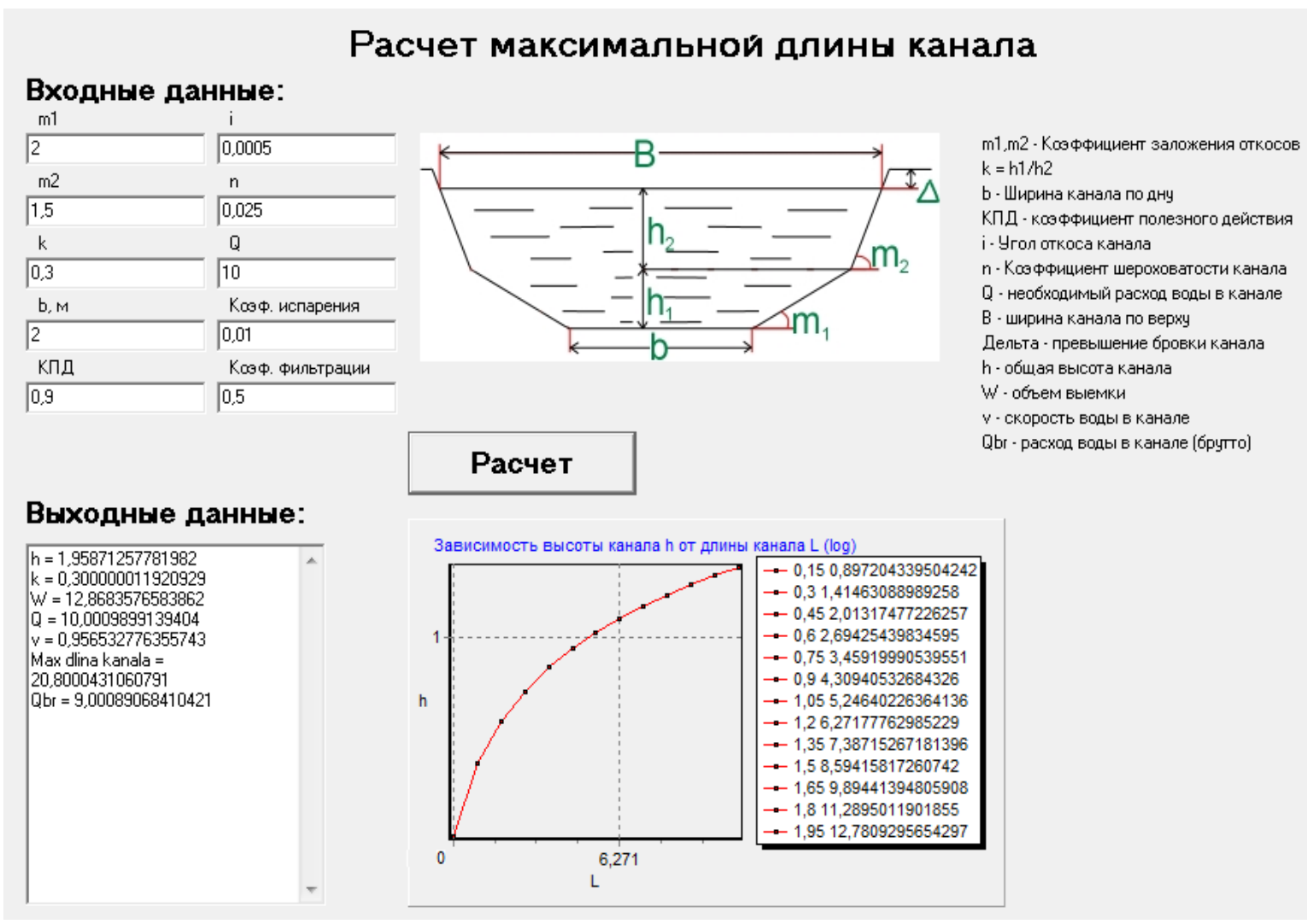

Рисунок 1 - Интерфейс программы для расчета максимальной длины канала 
Результаты расчетов полученные с помощью данной программы частично внесены в таблицу 1 и часть данных изображены в качестве графиков на рисунке 3,4 .

Таблица 1 - Предельная длина естественного русла

\begin{tabular}{|c|c|c|c|c|c|c|c|c|c|c|c|c|}
\hline № & $\begin{array}{c}h, \\
\mathrm{M}\end{array}$ & $\begin{array}{c}k \\
\left(h_{1} / h_{2}\right)\end{array}$ & $\begin{array}{c}b, \\
\mathrm{M}\end{array}$ & $k_{\phi}$ & $n$ & $m_{l}$ & $m_{2}$ & $\begin{array}{c}\omega_{\text {в }}, \\
\mathrm{M}^{2}\end{array}$ & $\begin{array}{c}W, \\
\mathrm{~m}^{3}\end{array}$ & $\begin{array}{c}v, \\
\mathrm{M} / \mathrm{c}\end{array}$ & $\begin{array}{c}Q_{\text {норм.бр, }}, \mathrm{M}^{3} / \mathrm{c} \\
l_{\text {пр, }} \\
\text { Км }\end{array}$ \\
\hline 1 & 2 & 0,3 & 2 & 0,5 & 0,025 & 2 & 1,25 & 9 & 12,47 & 0,98 & 9 & 22,5 \\
\hline 2 & 2,06 & 0,3 & 2 & 0,5 & 0,025 & 2 & 1 & 8,6 & 12,09 & 0,99 & 9 & 24,5 \\
\hline 3 & 2,17 & 0,5 & 2 & 0,3 & 0,025 & 2 & 1,25 & 9,44 & 13,55 & 1 & 10 & 39,2 \\
\hline 4 & 1,49 & 0,5 & 2 & 0,3 & 0,025 & 2 & 1,25 & 7,4 & 8,3 & 0,83 & 5 & 24,8 \\
\hline 5 & 2,37 & 0,75 & 3 & 0,3 & 0,0225 & 1,75 & 1,25 & 11,44 & 19,63 & 1,25 & 18 & 57,9 \\
\hline 6 & 1,95 & 0,3 & 2 & 0,5 & 0,025 & 2 & 1,5 & 9,45 & 12,87 & 0,95 & 9 & 20,8 \\
\hline 7 & 1,48 & 0,3 & 2 & 0,5 & 0,025 & 2 & 1,5 & 7,64 & 8,44 & 0,82 & 4,95 & 14,0 \\
\hline 8 & 1,26 & 0,3 & 2 & 0,5 & 0,025 & 2 & 1 & 6,06 & 6,02 & 0,84 & 3,6 & 13,6 \\
\hline 9 & 2,55 & 0,2 & 2 & 0,5 & 0,0225 & 2 & 1,5 & 11,71 & 19,91 & 1,23 & 18 & 33,7 \\
\hline 10 & 1,48 & 0,5 & 2 & 0,5 & 0,0225 & 3 & 1,5 & 6,78 & 7,48 & 0,83 & 4,5 & 15,2 \\
\hline 11 & 0,57 & 0,2 & 2 & 0,5 & 0,02 & 3 & 1,5 & 4,33 & 2,70 & 0,55 & 0,9 & 4,4 \\
\hline 12 & 1,40 & 0,75 & 1,5 & 0,3 & 0,225 & 1,75 & 1,25 & 6,46 & 6,56 & 0,86 & 4 & 22,9 \\
\hline 13 & 2,77 & 0,6 & 3,5 & 0,3 & 0,0225 & 3 & 1,5 & 13,26 & 26,57 & 1,38 & 27 & 75,1 \\
\hline 14 & 2,36 & 0,4 & 3 & 0,3 & 0,0225 & 3 & 1,5 & 13,34 & 24,27 & 1,28 & 22,5 & 59,4 \\
\hline 15 & 0,72 & 0,4 & 1 & 0,3 & 0,0225 & 3 & 1,5 & 5,33 & 4,28 & 0,70 & 2 & 13,3 \\
\hline 16 & 0,65 & 1 & 1,5 & 0,5 & 0,0225 & 2,5 & 1,75 & 4,57 & 2,93 & 0,57 & 1 & 4,5 \\
\hline 17 & 0,76 & 1 & 1,5 & 0,5 & 0,0225 & 1,25 & 1,25 & 3,92 & 2,6 & 0,6 & 1 & 5,7 \\
\hline 18 & 1,59 & 1 & 1,5 & 0,5 & 0,0225 & 1,5 & 1,5 & 7,25 & 7,82 & 0,9 & 5 & 15,3 \\
\hline 19 & 2,04 & 1 & 0 & 0,5 & 0,0225 & 1,5 & 1,5 & 7,35 & 7,81 & 0,9 & 5 & 15,7 \\
\hline 20 & 1,49 & 1 & 1,5 & 0,5 & 0,0225 & 2 & 1 & 6,92 & 7,60 & 0,91 & 5 & 16,1 \\
\hline 21 & 2,47 & 1 & 2 & 0,5 & 0,0225 & 1,5 & 1,5 & 10,92 & 17,32 & 1,18 & 15 & 30,6 \\
\hline 22 & 3,08 & 1 & 0 & 0,5 & 0,0225 & 1,5 & 1,5 & 11,10 & 17,35 & 1,17 & 15 & 31,2 \\
\hline 23 & 2,30 & 1 & 2 & 0,5 & 0,0225 & 2 & 1 & 10,39 & 16,82 & 1,20 & 15 & 32,4 \\
\hline
\end{tabular}

Как видно из таблицы 1 , если брать коэффициент откосов $\mathrm{m}_{1}=\mathrm{m}_{2}$, то у нас получается трапецеидальный канал, который уступает в ряде параметров каналу с полигональным сечением с аналогичными параметрами.

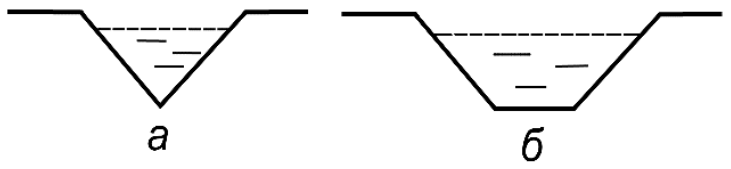

Рисунок 2 -

а) треугольное сечение канала;

б) трапецеидальное сечение ка-
Кроме того полигональный канал максимально близко обладает конфигурацией равноустойчивых откосов, что является значительным плюсом при рассмотрении вопроса оптимальности всего периметра канала на прочность. Также видно, что скорость течения в полигональном 
канале выше, чем скорость течения в трапецеидальном канале, как следствие, предельная длина канала тоже выше. Это касается также треугольного сечения канала. Сечения данных типов каналов показаны на рис.2
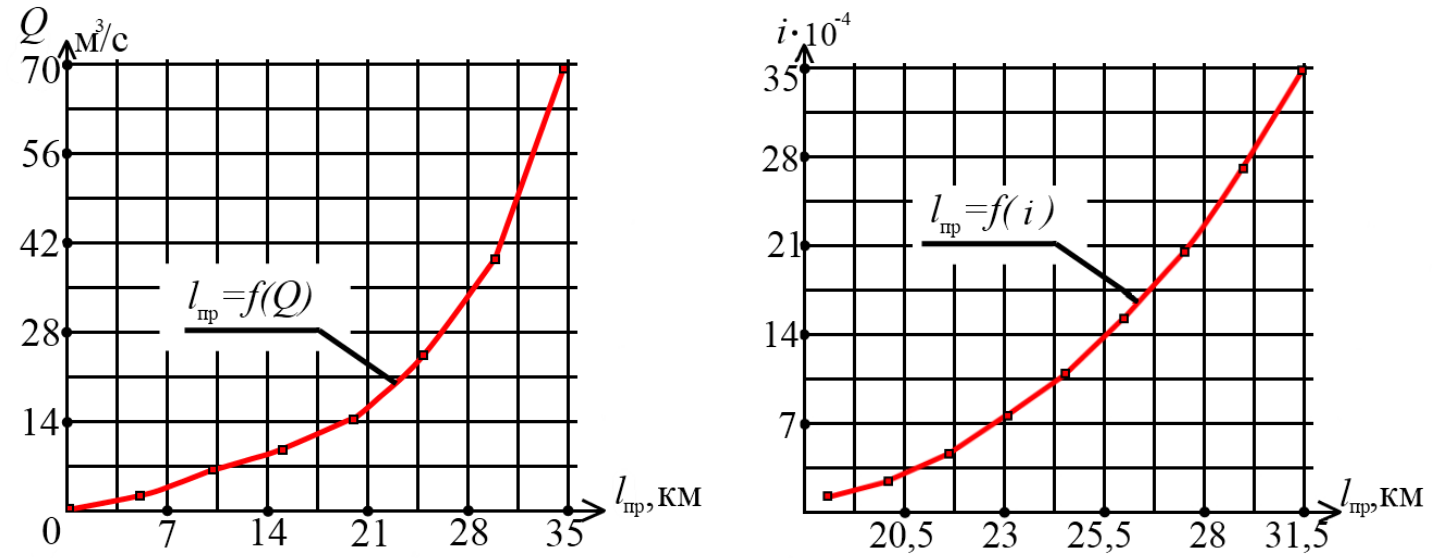

Рисунок 3 - Зависимость $l_{\text {пр }}=f(Q) \quad$ Рисунок $4-$ Зависимость $l_{\text {пр }}=f(i)$

Анализируя рисунок 3 можно заметить, что при последующем увеличении расхода воды в канале $Q$ показатель предельной длины канала $l_{\text {пр }}$ увеличивается уже значительно медленнее, в связи с тем, что, чем длиннее трасса по которой проходит вода, тем больше воды фильтруется, так как увеличивается поглащаемая площадь, а также с большей поверхности испаряется большее количество воды. Исходя из графика является возможным постановить следующее: при заданных параметрах наиболее эффективным будет расход воды в канале до 20м $3 /$. Однако брать данный расход как норматив не следует, в связи с тем, что при других параметрах канала полигонального сечения возможно график будет отличным от настоящего.

Сопоставляя данные таблицы 1 следует заметить что увеличение скорости воды в канале уменьшает обьъем выемки грунта, что в свою очередь уменьшает затраты на ряд работ. На рисунке 4 изображен график зависимости уклона $i$ к длине $l_{\text {пр. }}$ Отметим что значение уклона $i$ влияет на скорость течения воды в канале, а в свою очередь скорость течения воды в канале не должна вызывать размыва русла, т.е. не должна превышать «предельную скорость по размыву», значение которой характеризуется качественными характеристиками грунта в канале.

Рассматриваемая задача имеет степень многофакторной и сложной задачи в виду того, что искомая длина канала зависит от ряда параметров:

$$
l_{\text {пр }}=f\left(Q, b, h, m_{1}, m_{2}, n, i, k_{\phi}, E_{b}, \text { еидр. }\right)
$$

В (4) длина канала зависит от 10 и более параметров, которые в свою очередь варьируются для получения более точных результатов модели 
канала. Если каждый параметр варьировать хотя бы 4 значениями, то в целом количество всевозможных вариаций будет равно $4^{10}=1048576$. При помощи ручного счета такое количество расчетов, с последующим анализом, сделать невозможно или крайне сложно. Порой для счета большего количества значений для варьирования, а также большего количества варьируемых факторов, недостаточны даже современные ЭВМ. В связи с этим необходимо пользоваться методами математического планирования эксперимента [7]. Существует большое количество планов для математического планирования, наиболее подходящий план выбирается интуитивно, а также, следуя рекомендациям из $[7,8]$. Количество вариаций различных параметров может быть различным, в связи с тем, что некоторые параметры влияют значительно на функцию, а некоторые менее значительно. В связи с этим выбран план, удовлетворяющий требованию: $2^{2} / 4^{7} / 8^{1} / / 32$, где $2^{2}$ - это по 2 варьирования двух факторов, $4^{7}$ - это по 4 варьирования 7 факторов и $8^{1}$ - по 8 варьирований одного фактора, 32 - количество опытов. Выбранный план эксперимента для 10 факторов представлен в таблице 2.

Таблица 2 - План эксперимента для искомой величины $l_{n p}$.

\begin{tabular}{|c|c|c|c|c|c|c|c|c|c|c|c|}
\hline \multirow[b]{2}{*}{$\begin{array}{c}\text { № } \\
\text { опыта }\end{array}$} & \multicolumn{11}{|c|}{ Факторы } \\
\hline & $m_{1}$ & $m_{2}$ & $\begin{array}{c}k \\
\left(h_{1} / h_{2}\right)\end{array}$ & $\begin{array}{l}b, \\
\text { м }\end{array}$ & $E_{\tilde{\sigma}}$ & $i$ & $n$ & $Q$ & $\begin{array}{c}e, \\
\mathrm{M} / \text { сут }\end{array}$ & $\begin{array}{c}k_{\phi}, \\
\text { M/cyт }\end{array}$ & $\begin{array}{c}l_{n p}, \\
\mathrm{M}\end{array}$ \\
\hline 1 & 1 & 1 & 0.2 & 1 & 0.7 & 0.0003 & 0.015 & 3 & 0.01 & 0.05 & 513905 \\
\hline 2 & 1.5 & 1.5 & 5 & 3 & 0.77 & .0005 & 0.03 & 3 & 0.01 & 0.2 & 57482 \\
\hline 3 & 2 & 2 & 1 & 10 & 0.84 & .0007 & 0.015 & 3 & 0.02 & 1 & 4279 \\
\hline 4 & 2.5 & 2.5 & 2 & 6 & 0.9 & 0.001 & 0.03 & 3 & 0.0 & 5 & 625 \\
\hline 5 & 1.5 & 1 & 0.2 & 6 & 0.84 & 0.001 & 0.015 & 5 & 0.02 & 0.2 & 55774 \\
\hline 6 & 1 & 1.5 & 0.5 & 10 & 0.9 & 0.0007 & 0.03 & 5 & \begin{tabular}{|l|}
0.02 \\
\end{tabular} & 0.05 & 79769 \\
\hline 7 & 2.5 & 2 & 1 & 3 & 0.7 & 0.0005 & 0.015 & 5 & 0.01 & 5 & 4717 \\
\hline 8 & 2 & 2.5 & 2 & 1 & 0.77 & 0003 & & 5 & 0.0 & 1 & 12756 \\
\hline 9 & 1.5 & 1.5 & 1 & 6 & 0.9 & .0003 & 0.015 & 7 & \begin{tabular}{|l|} 
\\
\end{tabular} & 1 & 7380 \\
\hline 10 & 1 & 1 & 2 & 10 & 0.84 & 0.0005 & 0.03 & 7 & 0.01 & 5 & 1894 \\
\hline 11 & 2.5 & 2.5 & 0.2 & 3 & 0.77 & 0.0007 & 0.015 & 7 & 0.02 & 0.05 & 420693 \\
\hline 12 & 2 & 2 & 0.5 & 1 & 0.7 & 0.001 & 0.03 & 7 & 0.02 & 0.2 & 146700 \\
\hline 13 & 1 & 1.5 & 1 & - & 0.77 & 0.001 & 0.015 & 10 & 0.02 & 5 & 8471 \\
\hline 14 & 1.5 & 1 & 2 & 5 & 0.7 & 0.0007 & 0.03 & 10 & 0.02 & 1 & 41744 \\
\hline 15 & 2 & 2.5 & 02 & 10 & 0.9 & 0.0005 & 0.015 & 10 & $\mid 0.01$ & 0.2 & 34408 \\
\hline 16 & 2.5 & 2 & 0.5 & 6 & 0.77 & 0.0003 & 0.03 & 10 & 0.01 & 0.05 & 366091 \\
\hline 17 & 2 & 1 & 0.5 & 3 & 0.9 & 0.0003 & 0.015 & 12 & 0.02 & 5 & 2943 \\
\hline 18 & 2.5 & 1.5 & 0.2 & 1 & 0.84 & 0.0005 & 0.03 & 12 & \begin{tabular}{|l|}
0.02 \\
\end{tabular} & 1 & 19876 \\
\hline 19 & 1 & 2 & 0 & 6 & 0.77 & 0.0007 & 0.015 & 12 & 0.01 & 0.2 & 164811 \\
\hline 20 & 1.5 & 2.5 & 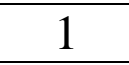 & 10 & 0.7 & 0.001 & 0.03 & 12 & 0.01 & 0.05 & 581796 \\
\hline
\end{tabular}




\begin{tabular}{|c|c|c|c|c|c|c|c|c|c|c|c|}
\hline 21 & 2.5 & 1 & 0.5 & 10 & 0.77 & 0.001 & 0.015 & 15 & 0.01 & 1 & 30309 \\
\hline 22 & 2 & 1.5 & 0.2 & 6 & 0.7 & 0.0007 & 0.03 & 15 & 0.01 & 5 & 8862 \\
\hline 23 & 1.5 & 2 & 2 & 1 & 0.9 & 0.0005 & 0.015 & 15 & 0.02 & 0.05 & 328786 \\
\hline 24 & 1 & 2.5 & 1 & 3 & 0.77 & 0.0003 & 0.03 & 15 & 0.02 & 0.2 & 131084 \\
\hline 25 & 2.5 & 1.5 & 2 & 10 & 0.7 & 0.0003 & 0.015 & 17 & 0.02 & 0.2 & 193653 \\
\hline 26 & 2 & 1 & 1 & 6 & 0.77 & 0.0005 & 0.03 & 17 & 0.02 & 0.05 & 690358 \\
\hline 27 & 1.5 & 2.5 & 0.5 & 1 & 0.84 & 0.0007 & 0.015 & 17 & 0.01 & 5 & 5335 \\
\hline 28 & 1 & 2 & 0.2 & 3 & 0.9 & 0.001 & 0.03 & 17 & 0.01 & 1 & 14393 \\
\hline 29 & 2 & 1.5 & 2 & 3 & 0.84 & 0.001 & 0.015 & 20 & 0.01 & 0.05 & 708367 \\
\hline 30 & 2.5 & 1 & 1 & 1 & 0.9 & 0.0007 & 0.03 & 20 & 0.01 & 0.2 & 89933 \\
\hline 31 & 1 & 2.5 & 0.5 & 6 & 0.7 & 0.0005 & 0.015 & 20 & 0.02 & 1 & 54696 \\
\hline 32 & 1.5 & 2 & 0.2 & 10 & 0.77 & 0.0003 & 0.03 & 20 & 0.02 & 5 & 5496 \\
\hline
\end{tabular}

Как видно из таблицы 2 факторы имеют некоторый диапазон и различное для некоторых факторов количество вариаций:

- 2 уровня для:e $=0,01 ; 0,02(\mathrm{M} / \mathrm{cyт})$;

$n=0,015 ; 0,03$;

- 8 уровней для: $Q=3,0 ; 5,0 ; 7,0 ; 10,0 ; 12,0 ; 15,0 ; 17,0 ; 20,0\left(\mathrm{~m}^{3} / \mathrm{c}\right)$.

- 4 уровня для: $m_{1}=1 ; 1,5 ; 2 ; 2,5$;

$m_{2}=1 ; 1,5 ; 2 ; 2,5$;

$k\left(h_{1} / h_{2}\right)=0.2 ; 0.5 ; 1 ; 2$.

$i=0,0001 ; 0,0004 ; 0,0007 ; 0,001$;

$k_{\phi}=0,05 ; 0,2 ; 1 ; 5$ (м/сут);

$E_{\mathrm{b}}=0,7 ; 0,77 ; 0,84 ; 0,9$;

$b=1 ; 3 ; 6 ; 10$ (м).

Зачастую одни параметры влиют более существенно на модель, в свою очередь другие же факторы влияют в меньшей степени на ту же модель, в связи с этим, интуитивно берем большее число варьирований для наиболее влиятельных факторов. В виду громадной трудоемкости решения подобных матриц размером 10х32, разработана программа для автоматического подсчета искомой формулы зависимости функции $l_{n p}$, от различных факторов. Результатом программы явилась следующая зависимость:

$$
\begin{gathered}
l_{n p}=925,115 \cdot m_{1}^{-0,1222} \cdot m_{2}^{-0,2854} \cdot k^{-0,01292} \cdot b^{-0,2097} \cdot E_{b}^{-5,4866} . \\
\cdot i^{0,095764} \cdot n^{-0,2926} \cdot Q^{0,73093} \cdot e^{-0,03647} \cdot k_{\phi}^{-1,0014}
\end{gathered}
$$

Результаты модели показывают зависимость параметров к искомой длине канала, в результате чего, одни параметры влияют более существенно относительно других. Ниже приведена зависимость влияния параметров на предельную протяженность длины русла в порядке возрастания: 


$$
k<e<m_{1}<i<m_{2}<n<b<Q<E_{b}<k_{\phi}
$$

Выводы. В результате проделанной работы получена модель (5) которая отражает отношение зависимости большого количества параметров гидравлического канала к искомой предельной длине канала. В ходе работы разработан программный комплекс, без помощи которого процесс получения модели был бы крайне сложным, а при задачах большего количества параметров и большего количества варьирований этих параметров, вовсе невозможным. Данный программный комплекс можно принять как дальнейшее развитие систем автоматизированного проектирования гидравлических объектов. Предложенная зависимость (5) также является вспомогательным средством процесса проектирования гидравлических каналов полигонального сечения.

\section{Библиографический список:}

1. Учинчус А.А. Гидравлические и технико-экономические расчеты каналов. М.: Изд-во лит-ры по строит-ву, 1965. -274 с.

2. СНиП 2.06.03-85 Мелиоративные системы и сооружения. - М.: ЦИТП Госстроя СССР, 1986. - 65 с.

3. Дементьев В.Г. Орошение. - М.: Колос, 1979. - 303 с.

4. Алибеков А.К., Горшков В. В. К вопросу оптимизации параметров каналов // Обеспечение охраны, улучшения и восстановления поверхностных водных объектов в Западно-Каспийском бассейновом округе: Сб.статей межрегион. науч.-практ. конференции. - Пятигорск: Западно-Каспийское бассейновое водное управление, 2011. - С. $179-182$.

5. Архангельский А.Я. Программирование в С++ Builder. 7-е изд. - М.: ООО «Бином-Пресс», 2010 896с. (1230с.): ил.

6. Корсаков С.М. Учебно-методический комплекс по дисциплине «Гидравлика». Нижний Новгород 2011. - 127с.

7. Налимов В.В., Голикова Т. И. Логические основания планирования эксперимента. - М.: Металлургия, 1981. - 150 с.

8. Таблицы планов эксперимента: для факторных и полиноминальных моделей/ Под. ред. Налимова В.В. - М.: Металлургия, 1982. - 752 с. 